\title{
Parameters of white LEDs respecting operational conditions
}

\begin{abstract}
Paper brings knowledge from measurement on white power LEDs for general lighting applications considering operational conditions that affect quantitative and qualitative light parameters of LEDs as well as basic electrical parameters. These data are necessary for correct design of luminaire and even for basic understanding of $L E D$ behaviour in real applications, so it can be used for creation of proper model of $L E D$ parameters degradation. Measuring of LED parameter changes is based on spectroradiometry to provide information not only about luminous flux change but also about qualitative parameters changes such as chromaticity temperature and colour rendering index.
\end{abstract}

Keywords: LED, degradation, temperature, current, spectral distribution, supply voltage

\section{Introduction}

Using of white LEDs (Light Emitting Diodes) for general lighting is on rise. It is caused by several factors continuously decreasing prices of LEDs, variable construction designs of luminaires, LED luminaires are suitable for implementation to advanced lighting systems due to their good controllability and others. But this rise brings new challenges for both researchers and manufacturers in the area of understanding the behaviour of LED parameters under real operating conditions. Current state of light parameters stability research is mostly focused on decrease of luminous flux during lifetime and influence of junction temperature on luminous flux. However using white LEDs especially for general lighting is conditioned by fulfilling of other parameter limits - operating voltage, luminous efficacy, colour rendering index, chromaticity temperature. Therefore the data based on spectral distribution changes of white LEDs respecting possible operational conditions are much more convenient for evaluation of possible influences on LED light sources.

\section{Temperature}

Temperature is key factor of LED operation. Recommended limits of junction temperature are given by most manufacturers and it is up to the manufacturer of the luminaire to keep the temperature in suitable range. Lower junction temperature generally brings better performance of luminous flux and thus better efficiency. However the possibilities of cooling are not infinite and each luminaire has to meet also other requirements - price, dimensions, weight, supply power etc. Considering commonly recommended operational junction temperatures below $90{ }^{\circ} \mathrm{C}$, significant factor for junction temperature stabilizing is also the ambient temperature of such luminaire.

Other significant factor is the thermal resistance of the whole lighting device. This quantity depends on LED construction, its mounting and thermal way of the whole LED package to the heatsink and it is also provided by the LED package manufacturer. With using the thermal resistance coefficient the junction temperature can be calculated as follows [1]:

$$
T_{j}=T_{b}+P \cdot R_{j-b},
$$

where $T_{j}$ is junction temperature $\left({ }^{\circ} \mathrm{C}\right), T_{b}$ is mounting board temperature $\left({ }^{\circ} \mathrm{C}\right), P$ is supplied power $(\mathrm{W})$ and $R_{j-b}$ is thermal resistance between junction and mounting board $\left({ }^{\circ} \mathrm{C} \cdot \mathrm{W}^{-1}\right)$.

This parameter is of course variable among different LEDs, but more significant issue for temperature influence measurement is the stability of this parameter at different operating conditions. According to research focused on thermal management of LEDs with different attach materials [2] this parameter seems to be almost constant in common temperature range of heatsink $\left(20{ }^{\circ} \mathrm{C}-90{ }^{\circ} \mathrm{C}\right)$. There are also other options, how the junction temperature can be determined, more or less accurate [3], but study of junction temperature determination is not an issue of this paper.

\section{Measurement conditions}

Measuring samples were chosen with regards to provide sufficient power, luminous efficacy with respect to favourable price, so briefly with respect to parameters that are important for many luminaire manufacturers. LED used at this measurement were all of the same type - Vigan GTP03W54101140 connected on PCB (printed circuit board) hexagon plate with following specification:

$\begin{array}{lll}\text { - } & \text { luminous flux: } & 140-150 \mathrm{Im} \\ \text { - } & \text { chromaticity temperature: } & 5500-6000 \mathrm{~K} \\ \text { - } & \text { colour rendering index: } & 60-90 \\ \text { - } & \text { operating voltage: } & 3.0-3.6 \mathrm{~V} \\ \text { - } & \text { thermal resistance: } & 350 \mathrm{~mA} \\ \text { - } & \text { operating temperature: } & -40 \mathrm{~K} \cdot \mathrm{W}-1 \\ \text { - } & \text { thermal-voltage coefficient: } & -5 \mathrm{mV} \cdot{ }^{\circ} \mathrm{C}^{-1} \mathrm{C}\end{array}$

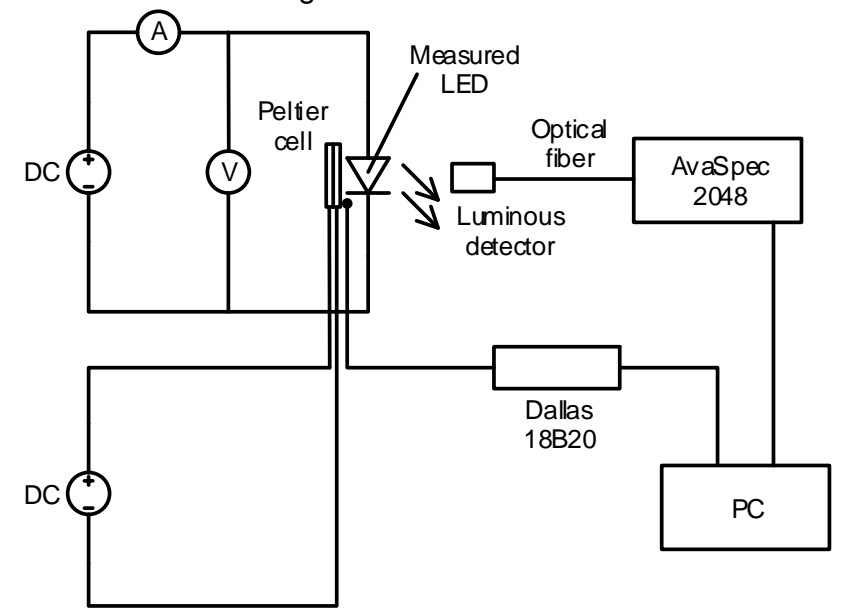

Fig. 1. Wiring scheme

Measurement of parameter modification was performed in lighting laboratory at Brno University of Technology. Cooling device used for temperature control at the measurement was based on Peltier effect. Peltier cell supplied with DC current and attached to cooler that consists of aluminium heatsink and fan of sufficient airflow power is used for both cooling and eventual heating of the measured LED. Fan is used for enabling of sufficient cooling power and using of aluminium heatsink with sufficient mass provides temporary stability of the set temperature. LED is connected 
to the Peltier cell with using of thermal grease, its thermal resistance $\left(R_{t g}=2.5 \mathrm{mK} \cdot \mathrm{W}^{-1}\right)$ is negligible in comparison with thermal resistance of LED package (at this measurement $R_{j-b}=12 \mathrm{~K} \cdot \mathrm{W}^{-1}$ ) for enabling best possible performance of temperature control. Measuring of LED board temperature is performed with thermocouple Dallas 18B20 (12 bit, accuracy $\pm 0.5^{\circ} \mathrm{C}$, measuring range $-55^{\circ} \mathrm{C}-125^{\circ} \mathrm{C}$ ). LED is supplied with stable current source Keithley $2601 \mathrm{~b}$ that is used both as power source and voltage/power meter. And finally the light parameters are measured with spectroradiometric system Avantes AvaSpec-2048 with optical fiber Avantes FC-UV200-2.

\section{Luminous flux change}

Luminous flux is commonly observed parameter and it is also often presented directly in the datasheet of LED manufactures. The degradation curve is supposed to be of descending character with increased junction temperature, mainly caused by increasing number of non-radiative recombination in $\mathrm{PN}$ junction [4].

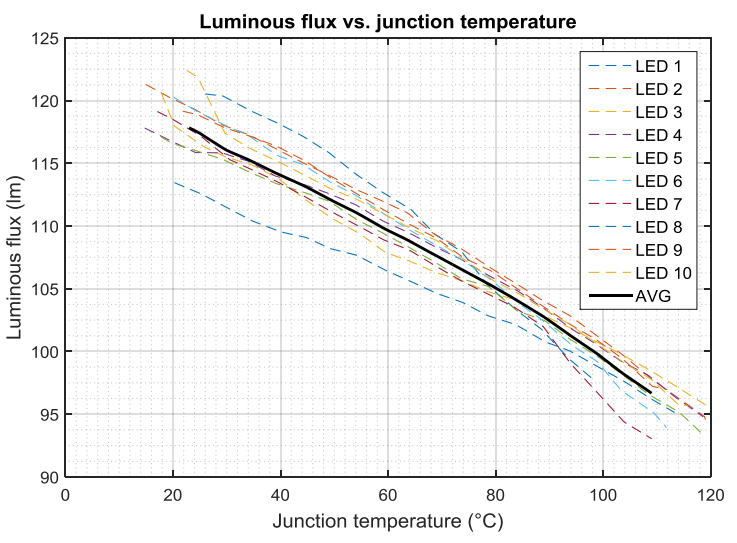

Fig. 2. Luminous flux degradation at different $\mathrm{PN}$ junction temperatures

As presented in fig. 2. the degradation of luminous flux measured at tested LEDs was nearly linear till approximately $90{ }^{\circ} \mathrm{C}$ junction temperature with the rate of $-0.24 \mathrm{Im}^{\circ} \mathrm{C}^{-1}$. Above this temperature the decrease is becoming slightly faster $\left(-0.28 \mathrm{Im} \cdot{ }^{\circ} \mathrm{C}^{-1}\right)$.

Considering this luminous flux decrease it does not seem very important to preserve stable operating temperature of the PN junction - the difference between well-cooled LED (junction temperature $50{ }^{\circ} \mathrm{C}$ ) and LED with commonly considered high temperature $\left(100^{\circ} \mathrm{C}\right)$ is at luminous flux only approximately $12 \%$. This value seems to be quite low especially if considered the price of well-designed heatsink for luminaire intended for general lighting purposes.

\section{Spectral distribution change}

Spectral distribution of emitted light is crucial factor that affects all parameters observed at LED, both quantitative and qualitative. Spectral distribution (dominant wavelength peak respectively) of monochromatic LEDs should stay stable at all operational conditions, but even that is not always valid due to the fact of bandgap lowering under thermal and electric stress that can cause so called spectral shift [5].

Degradation of secondary emitting peak (emitted by phosphor coating) can be partially eliminated by using of remote phosphor coating. If designed properly this solution may prevent the phosphor from being influenced by the heat produced in the PN junction - but this solution has also significant disadvantages: high price, high demands on manufacture process accuracy and at current state of manufacturing also higher consumption of phosphor material compared to design with direct phosphor coating [6].
However white LED spectral distribution can be affected even if no spectral shift occurs. White LEDs based on using of blue LED with phosphor coating are depending also on change of dominant peak ratio that determines chromaticity temperature and other qualitative parameters. Determining the factors that affect this ratio is complex issue, because each factor is based on different physical process in LED change on junction level, change of phosphor coating, change of electrical parameters of metal contacts and others [3].

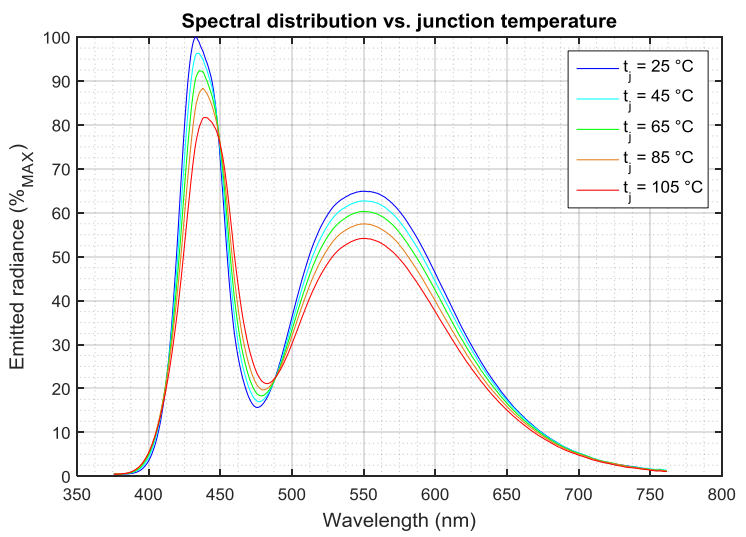

Fig. 3. Spectral distribution modification at different PN junction temperatures

Measured spectral distribution of emitted light provides some important data. At first there can be spotted different decrease of emitted energy on different wavelengths (frequencies). Higher value of energy decrease can be observed at dominant peak in the blue range of spectrum. If compared with the energy drop of side peak (emitted by phosphor) it is obvious that high junction temperature influences more significantly the primary light generation in PN junction than the secondary light generation in phosphor coating. Moreover higher junction temperature would probably increase this difference of these two energy drops due to place of heat origin, which is situated in the PN layers.

Other important fact lies in visible spectral shift of primary (dominant) peak of LED. Measured spectral shift moved dominant wavelength from $444 \mathrm{~nm}$ at $25{ }^{\circ} \mathrm{C}$ to $451 \mathrm{~nm}$ at $105^{\circ} \mathrm{C}$ (PN junction temperature). It proves right the above mentioned idea of lowering the bandgap under thermal stress. This bandgap change influences the primary light emission and it has consequent impact on other qualitative parameters. Secondary emission peak wavelength remains stable at all operational temperatures, so the stability of phosphor emission stays unchangeable. Key point for stabilizing the spectral distribution of white LEDs is then based in performing the spectral stability of PN junction emission at different operational temperatures. At this point it also seems to be counterproductive to use the remote phosphor design, because from the view of spectral distribution it will only increase the rate of spectral instability.

\section{Chromaticity temperature and colour rendering index}

Chromaticity temperature (CT) is a qualitative parameter that is important from the view of luminaire design. Its stability should be fixed to designed value at all operational states, nevertheless if spectral distribution of emitted light changes chromaticity temperature also changes its value. Similar change can occur at Colour rendering index (CRI). These parameter changes can cause a significant issue, especially if considered how toughly these parameters are achieved at luminaires equipped with LED sources. 


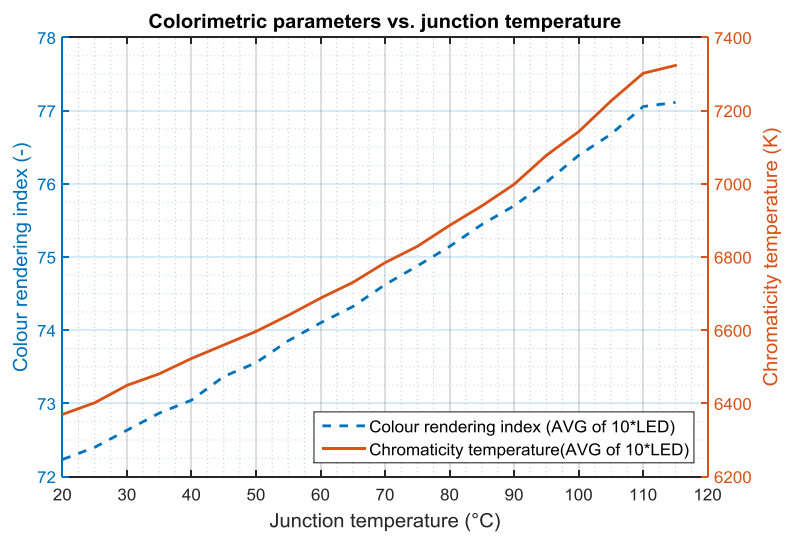

Fig. 4. Increase of Colour rendering index and Chromaticity temperature at different $\mathrm{PN}$ junction temperatures

Instability of spectral distribution of emitted light influences qualitative parameters quite significantly, as presented in Fig. 4. Change of CRI may not be very troubling, especially if considered its increasing characteristics - with increased temperature better results of $\mathrm{CRI}$ can be obtained.

Much more troubling issue for stabilizing the luminous parameters in real luminaires brings change of the CT. The rate of the change about $1000 \mathrm{~K}$ becomes significant not only for high demand lighting systems, but it can be problem also for common use.

Stabilizing quality parameters at commonly used LEDs is at current situation possible only by using of sufficiently dimensioned heatsinks with high heat capacity, but it is quite questionable issue. At first there is high price of such design, moreover this solution brings long stabilization period that takes away one of key advantages of using LEDs - quick response after switching on, because thermal stabilizing would take enormous time period, it can reach one hour or even more.

\section{Electric parameters}

Using of accurate direct current source brings the possibility of measuring the changes of supply voltage. This parameter should be dependent on junction temperature according to thermal-voltage coefficient that is often presented by the LED manufacturer. As presented in measuring conditions chapter this coefficient is usually negative, so with increasing junction temperature the supply voltage value decreases, which corresponds with decrease of consumed power. Stability of this coefficient is very important due to significance of forward voltage on the field of junction temperature determination - one of most often used method evaluates junction temperature according to forward voltage drop.

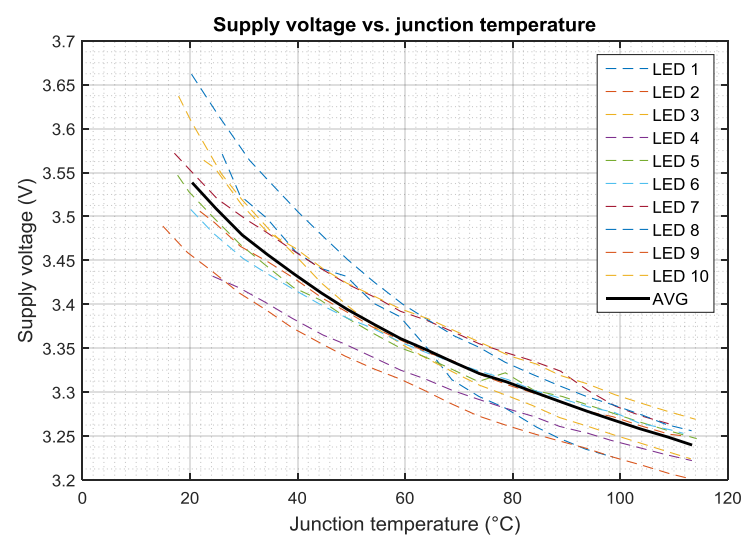

Fig. 5. Supply voltage change depending on $\mathrm{PN}$ junction temperature
Using of constant thermal-voltage coefficient seems to be inappropriate as presented in fig. 5. Especially in the range of lower junction temperatures this coefficient is not a constant value - rate of voltage decrease within the temperature raise is of decreasing character: above the junction temperature of $60{ }^{\circ} \mathrm{C}$ the thermal-voltage coefficient is becoming almost constant with the value about - $2.4 \mathrm{mV} \cdot{ }^{\circ} \mathrm{C}^{-1}$. Designed value of $-5 \mathrm{mV} \cdot{ }^{\circ} \mathrm{C}^{-1}$ can be achieved only at very low junction temperatures below $40{ }^{\circ} \mathrm{C}$ that is almost impossible at general lighting luminaires.

Change of consumed power value has also to be put under proper research. Because it is not corresponding linearly with the decrease of luminous flux, it is obvious that also the decrease of luminous efficacy is not linear at all operational temperatures of $\mathrm{PN}$ junction and it can help with optimizing of heatsink to provide most convenient operation of LED from different points of view.

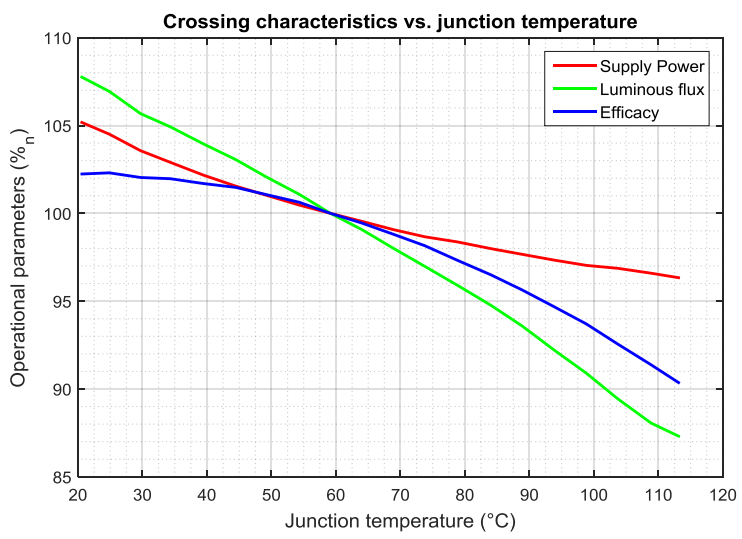

Fig. 6. Operational parameters depending on $\mathrm{PN}$ junction temperature - nominal temperature set to $60^{\circ} \mathrm{C}$

Decreasing character of supply voltage within the use of stabilized current provides a non-linear characteristic of consumed power, which has positive effect on luminous efficacy degradation within the rise of junction temperature. According to the luminous efficacy the best results for efficient lighting can be achieved in the range of junction temperatures between $40^{\circ} \mathrm{C}$ and $70^{\circ} \mathrm{C}$. Critical temperature for decrease of efficiency is above $90^{\circ} \mathrm{C}$ which is also critical value for preventing LED from damage and thermal impacts on the chip and its package and consequent shortening of LED operation lifetime.

\section{Conclusion}

Crucial factor for proper, safe and long-life operation of LED is thermal management. As presented in this paper the temperature of chip, resp. PN junction temperature has significant impact on all operational parameters of LED, both luminous and electric ones.

Degradation of luminous flux is only partial task of LED parameter stability research. Much more convenient method for parameter evaluation is based on research of spectral distribution of emitted light that involves temperature dependence of quantitative (luminous flux) and qualitative (CRI, CT) luminous parameters. As presented quite marginal changes of spectral distribution ratio and dominant peak shift can cause quite significant changes of qualitative luminous parameters. This research is more important as LEDs are becoming basic light sources in general lighting systems. As presented above more important changes at some white LEDs from the view of light system design were observed at qualitative parameter then at commonly researched 
luminous flux, which degradation rate reached acceptable value.

Increasing PN junction temperature brings changes of supply voltage within its thermal-voltage coefficient that is not a constant value as commonly presented. Therefore it should be always interpreted as a function of temperature that can be reached at real operation. Decrease of supply voltage or supply power respectively has positive impact on electric efficiency of LEDs and it also supports correct determination of proper operational temperature range - for LEDs in this measurement $40^{\circ} \mathrm{C}-70^{\circ} \mathrm{C}$.

Excessively designed heatsink does not bring any significant contribution. It increases the price, it does not bring rapid increase of luminous flux and it can also cause degradation of qualitative luminous parameters. Moreover it causes long period of parameter stabilization that takes away one of most important advantages of LED usage - switchingon speed and speed of luminous output stabilization.

Similar questionable contribution bring improvements such as using of remote phosphor that bring only partial increase of luminous flux, but they intensify the changes in spectral distribution of emitted light with consequent deterioration of qualitative luminous parameters.

\section{Acknowledgement}

This research work has been carried out in the Centre for Research and Utilization of Renewable Energy (CVVOZE). Authors gratefully acknowledge financial support from the Ministry of Education, Youth and Sports of the Czech Republic under NPU I programme (project No. LO1210) and BUT specific research programme (project No. FEKT-S-142520).

At this place authors would also like to thank Dr. Skoda from Brno University of Technology for consulting the results of performed measurements.

\section{REFERENCES}

[1] Xiang-you Lu, Tse-Chao Hua, Mei-jing Liu, Yuan-xia Cheng, "Thermal analysis of loop heat pipe used for high-power LED," Thermochimica Acta, Volume 493, Issues 1-2, 10 September 2009, Pages 25-29, ISSN 0040-6031

[2] You, J.P.; He, Y.; Shi, F.G., "Thermal management of high power LEDs: Impact of die attach materials," Microsystems, Packaging, Assembly and Circuits Technology, 2007. IMPACT 2007. International , vol., no., pp.239,242, 1-3 Oct. 2007

[3] Pavelka, T.; Ptacek, M. "Degradation of LED parameters during operation: Brief review of currently used models and measuring methods." In Proceedings of the 2015 16th International Scientific Conference on Electric Power Engineering (EPE). Ostrava, Czech Republic: VŠB-TU Ostrava, 2015. pp. 523-526. ISBN: 978-1-4673-6787-5.

[4] Li, J., M. et al. "Thermal Management for LED Applications," Springer Science+Business Media, New York, 2014, pp. 39 42, ISBN: 978-1-4614-5091-7.

[5] Senawiratne, J., et al. "Junction temperature, spectral shift, and efficiency in GalnN-based blue and green light emitting diodes." Thin Solid Films 518.6 (2010): 1732-1736.

[6] Mingzhi, D., Jia, W., Huaiyu, Y., Cadmus, Y., Kouchi, Z. "Thermal analysis of remote phosphor in LED modules." Journal of Semiconductors, 34(5), 2013, 053007.

\section{Authors:}

Tomáš Pavelka, Brno University of Technology, Czech Republic, e-mail:pavelkat@feec.vutbr.cz

Petr Doležal, Brno University of Technology, Czech Republic, e-mail:xdolez59@stud.feec.vutbr.cz

Pavel Sláma, Brno University of Technology, Czech Republic, e-mail:xslama18@stud.feec.vutbr.cz 